\section{Arbeitsgemeinschaft der Wissenschaftlichen Medizinischen Fachgesellschaften e.V.}

\author{
A. M Gressner ${ }^{1}$ und O. A Gressner ${ }^{2}$ \\ ${ }^{1}$ Labor Dr. Wisplinghoff Berlin, Berlin, Deutschland \\ ${ }^{2}$ Labor Dr. Wisplinghoff Köln, Köln, Deutschland
}

\section{Synonym(e) AWMF}

Definition Die AWMF ist ein eingetragener Verein, in dem die wissenschaftlichen Fachgesellschaften der Medizin zusammengeschlossen sind zum Zwecke der Förderung der fächerübergreifenden Zusammenarbeit ihrer Mitgliedsgesellschaften bei der Wahrnehmung wissenschaftlich-medizinischer Aufgaben und Ziele gegenüber den damit befassten (politischen) Institutionen und Entscheidungsträgern.

Beschreibung Die 1962 von damals 16 Gesellschaften auf Anregung der Deutschen Gesellschaft für Chirurgie in Frankfurt ins Leben gerufene AWMF ist ein eingetragener, gemeinnütziger Verein, dem derzeit 162 wissenschaftliche Fachgesellschaften aus allen Bereichen der Medizin angehören. Die AWMF vertritt Deutschland im Council for International Organizations of Medical Sciences (CIOMS) und verfolgt im Einzelnen folgende Aufgaben und Ziele:

- Erarbeitung grundsätzlicher und fächerübergreifender Empfehlungen und Resolutionen und Vertretung gegenüber staatlichen Institutionen und Körperschaften der Politik und ärztlicher Selbstverwaltung
- Vertretung der Interessen der medizinischen Wissenschaft nach außen

- Förderung einer leistungs- und zukunftsorientierten Weiterentwicklung der medizinischen Wissenschaften und der ärztlichen Praxis durch Zusammenarbeit mit der Bundesärztekammer, dem medizinischen Fakultätentag, der Gemeinschaft fachärztlicher Berufsverbände und den Einrichtungen der Wissenschaftsförderung

- Qualitätssicherung ärztlicher Berufsausübung

- Elektronische Publikation wissenschaftlicher Literatur

- Erstellung und Publikation von Leitlinien für Diagnostik und Therapie

Die AWMF-Aktivitäten werden getragen von der Delegiertenkonferenz, dem Präsidium und dem Präsidenten. Für die Bearbeitung besonderer Themen werden Kommissionen aus dem Kreis der Delegierten und gegebenenfalls durch Hinzuziehen Sachverständiger aus den Mitgliedsgesellschaften gebildet.

\section{Adresse}

AWMF-Geschäftsstelle

Ubierstr. 20

D-40223 Düsseldorf

Tel.: 021131282.

Fax: 021131681.

AWMF-Geschäftsstelle Berlin

Birkenstr. 67

D-10559 Berlin

Tel.: 03020097777

Internet: www.awmf.org 